REVISTA PROYECCIONES N ${ }^{\circ}$ 7: 19-59

JUNIO 1984 - I.S.S.N. 0716-0917.

\title{
EL MÉTODO DE RITZ PARA PROBLEMAS DE VALOR EN LA FRONTERA
}

OSCAR ROJO J.

RICARDO SOTO $M$.

LUIS VALDIVIA R.
(*)

(*)

\section{1.- INTRODUCCION.}

La ventajas computacionales que trae consigo el uso de funciones seccionalmente polinomiales en la aplicación del método de Ritz, para aproximar la solución de un problema de valor en la frontera, ha renovado el interés por dicho método. Para tal tipo de espacios de funcio nes, el método de Ritz y el método de elemento finito coinciden.

El presente trabajo tiene por propósito exponer teoremas bási cos de la formulación variacional de un problema de valor en la frontera, como también estudiar los teoremas fundamentales del método de Ritz. El trabajo termina con la aplicación de todo lo anterior a problemas elípti cos de segundo orden en una variable y en el plano.

(*) Departamento de Matemáticas, Facultad de Ciencias, Universidad del Norte. 
Supongamos que nuestro problema de valor en la frontera admite solución y que puede formularse en la forma:

Sean $X$ e $Y$ subespacios vectoriales de un espacio $Z$ con un producto interior real denotado por $(.,$.$) . Dado \mathrm{f} \in \mathrm{Y}$ y un operador lineal

$$
L: X \rightarrow Y,
$$

encontrar $\mathrm{u} \in \mathrm{x}$ tal que

$$
\mathrm{Lu}=\mathrm{f},
$$

donde el operador L satisface las propiedades

(1..2) $(L u, v)=(u, L v)$ para todo $u, v \in X$

(1.3) (Lu, u $)>0$ para todo $u \in X, u \neq 0$

es decir, I es un operador lineal simétrico y definido positivo.

Estas propiedades de L nos permiten concluir que (Lu, v) es un producto interior en $\mathrm{x}$.

El subespacio $\mathrm{X}$ contiene aquellas funciones para las cuales el operador l está definido y que satisfacen las condiciones de frontera.

Consideremos la siguiente funcional, por ahora definida sólo en $\mathrm{X}_{\text {, }}$

$$
J(u)=(L u, u)-2(f, u), u \in X
$$

Tenemos los siguientes teoremas:

Teorema 1.1.

Si $u \in X$ satisface lá ecuación $L u=f$ entonces

$$
J(u)=\min \{J(w): w \in x\}
$$

Demostración:

Sea $w \in X$. Tenemos

$$
\begin{aligned}
J(w)-J(u) & =(L w, w)-2(f, w)-(L u, u)+2(f, u) \\
& =(L w, w)-2(L u, w)-(L u, u)+2(L u, u) \\
& =(L w, w)-2(L u, w)+(L u, u) \\
& =(L w, w)-(L u, w)-(u, L w)+(L u, u)
\end{aligned}
$$




$$
\begin{aligned}
& =(L w-L u, w-u) \\
& =(L(w-u), w-u) \geqslant 0
\end{aligned}
$$

Teorema 1.2 .

$\mathrm{u} \in \mathrm{X}$ minimiza la funcional $\mathrm{J}$ en $\mathrm{X}$ si y sólo si

$$
(\mathrm{Lu}, \mathrm{v})=(\mathrm{f}, \mathrm{v}) \text { para todo } \mathrm{v} \in \mathrm{X}
$$

Demostración:

Supongamos que $u$ minimiza la funcional $J$ en $X$. Sea $v \in X Y$

$\alpha \in \mathbb{R}$. Tenemos,

$$
\begin{aligned}
J(u+\alpha v) & =(L u+\alpha L v, u+\alpha v)-2(f, u+\alpha v) \\
& =J(u)+\alpha^{2}(L v, v)+2 \alpha(L u, v)-2 \alpha(f, v)
\end{aligned}
$$

\section{Entonces,}

$T(\alpha)=J(u+\alpha v)-J(u)=\alpha^{2}(L v, v)+2 \alpha(L u, v)-2 \alpha(f, v)$

es una expresión cuadrática en $\alpha$ que alcanza un mínimo en $\alpha=0$ pues $T(\alpha) \geqslant 0$ para todo $\alpha$ real. Por 10 tanto, $T^{\prime}(0)=0$.

Este resultado implica que

$$
(\mathrm{Lu}, \mathrm{v})=(\mathrm{f}, \mathrm{v}) \text { para todo } \mathrm{v} \in \mathrm{X}
$$

Recíprocamente, supongamos ahora que (1.5) sea cierta. En par ticular, tenemos

$$
(\mathrm{Lu}, \mathrm{u})=(\mathrm{f}, \mathrm{u}) \text {. }
$$

Entonces, para cualquier $\mathrm{v} \in \mathrm{X}$ tenemos

$$
\begin{aligned}
J(v)-J(u) & =(L v, v)-2(f, v)-(L u, u)+2(f, u) \\
& =(L v, v)-2(L u, v)+(L u, u) \\
& =(L(v-u), v-u) \geqslant 0
\end{aligned}
$$

El método de Ritz busca una aproximación de $u \in X$, solución de $L u=f$, minimizando la funcional $J$ sobre un espacio finito dimensional $X_{N}$. Esto conduce a un sistema de ecuaciones lineales, cuya matriz de coeficientes es definida positiva, cualquiera sea la elección de la base para $\mathrm{X}_{\mathrm{N}}$. Tenemos en este punto un aspecto muy importante: esco- 
ger bases atractivas desde el punto de vista computacional, esto es, bases que conduzcan a una matriz de coeficientes esparcida y con cierta es tructura que facilite la resolución del sistema de ecuaciones lineales. Sin embargo, normalmente estas bases están dadas por funciones que no pertenecen a X. Felizmente esta dificultad puede ser superada extendien do adecuadamente $\mathrm{x}$ a un espacio $\mathrm{K}$. Más aún, esta extensión es tal que en $\mathrm{K}$ tenemos un producto interior a $(.,$.$) cuya restricción a \mathrm{X} \times \mathrm{K}$ satis face

(1.6) $\quad a(u, w)=(L u, w), \quad u \in x y$ we $k$.

Además, es posible definir para $w \in K$,

(1.7) $\quad J(w)=a(w, w)-2(f, w)$

\section{2.- FORMULACION VARIACIONAL.}

Suponemos que $\mathrm{K}$ es una extensión de $\mathrm{X}$, con un producto interior a(.,.) tal que (1.6) se cumple. Sea
(2.1)

$$
|| w||_{a}=\sqrt{a(w, w)}
$$
para $w \in K$,

la norma inducida por a $(.,$.$) .$

El espacio $K$ podría no ser un espacio completo respecto de la norma ||$\cdot \|_{a}$. Es interesante, desde el punto de vista teórico, trabajar en un espacio de Hilbert. Por esta razón, consideramos la completación de $\mathrm{K}$ respecto de la norma $\|.\|_{\mathrm{a}}$, que denotamos mediante $\mathrm{H}$. Entonces, para $w$ y $v \in H$ existen sucesiones de Cauchy $\left(w_{n}\right)$ y $\left(v_{n}\right)$ en $K$ tales que, por definición,

$$
\begin{aligned}
& \left\|w_{n}\right\|_{a} \longrightarrow \quad\|w\|_{a} \quad y \\
& \left\|v_{n}\right\|_{a} \longrightarrow\|v\|_{a}
\end{aligned}
$$

cuando $\mathrm{n} \rightarrow \infty$.

Además, tenemos

$$
\begin{aligned}
\left|a\left(w_{n}, v_{n}\right)-a\left(w_{m}, v_{m}\right)\right| & =\left|a\left(w_{n}-w_{m}, v_{n}\right)-a\left(w_{m}, v_{m}-v_{n}\right)\right| \\
\leqslant & || w_{n}-w_{m}||_{a}|| v_{n}||_{a}+|| w_{m}||_{a}|| v_{m}-v_{n}||_{a} .
\end{aligned}
$$


De esta desigualdad podemos concluir que $\left(a\left(w_{n}, v_{n}\right)\right)$ es una su cesión de Cauchy en $\mathbb{R} y$, por lo tanto, una sucesión convergente en $\mathbb{R}$. En tonces, podemos definir

$$
a(w, v)=\lim _{n \rightarrow \infty} a\left(w_{n}, v_{n}\right) \text { para } w, v \in H
$$

De este modo hemos podido extender el producto interior a $(.$, ) al espacio H. Así entonces $\mathrm{H}$ es un espacio de Hilbert. Deseamos ahora extender la funcional $\mathrm{J}$ definida en (1.7) al espacio H. Para ello es necesario definir adecuadamente el término ( $f, w)$ para $w \in H$. Existe una sucesión $\left(w_{n}\right)$ en $K$ tal que

$$
|| w_{n}-w_{m} \|_{a} \rightarrow 0 \text { cuando } n \rightarrow \infty y m \rightarrow \infty \text {. }
$$

Tenemos,

(2.2) $\left|\left(f, w_{n}\right)-\left(f, w_{m}\right)\right|=\left|\left(f, w_{n}-w_{m}\right)\right| \leqslant|| f||_{2}|| w_{n}-w_{m} \|_{2}$

donde $\|\cdot\|_{2}=\sqrt{(.,)}$.

Definición.

Decimos que a $(., \ldots)$ es elíptico en $\mathrm{K}$ si y sólo si existe $\alpha>0$ tal que

$$
a(w, w) \geqslant \alpha|| w||_{2}^{2} \text { para todo } w \in K .
$$

Con la introducción de esta definición, la desigualdad (2.2) puede escribirse como

$$
\left|\left(f, w_{n}\right)-\left(f, w_{m}\right)\right| \leqslant \frac{1}{\sqrt{a}}|| f||_{2}|| w_{n}-w_{m}||_{a} .
$$

Por 10 tanto, si a $(.,$.$) es elíptico en K$ entonces ( $\left(f, w_{n}\right)$ ) es una sucesión de Cauchy en $\mathbb{R} y$, por lo tanto, convergente. Así podemos definir 
Definición:

Para we H, definimos

1) $(f, w)=\lim _{n \rightarrow \infty}\left(f, w_{n}\right)$, donde $\left(w_{n}\right)$ es una sucesión de Cauchy en $k$ tal que $\lim _{n \rightarrow \infty}|| w_{n}-\left.w\right|_{a}=0$

2) $J(w)=a(w, w)-2(f, w)$

Tenemos el siguiente teorema:

Teorema 2.1.

$\mathrm{Si} S$ es un subespacio de $\mathrm{H}$, en particular $\mathrm{S}=\mathrm{H}$, entonces $J(u)=\min \{J(v): v \in s\}$

si y sólo si

$$
\mathrm{a}(\mathrm{u}, \mathrm{v})=(\mathrm{f}, \mathrm{v}) \text { para todo } \mathrm{v} \in \mathrm{S} \text {. }
$$

Demostración.

Totalmente análoga a la demostración del teorema 1.2, ahora $\operatorname{con}(L u, v)=a(u, v)$.

Corolario.

Si $u \in S, S$ subespacio de $H$, en particular $S=H$, es tal que $\mathrm{J}(\mathrm{u})=\min \{\mathrm{J}(\mathrm{v}): \mathrm{v} \in \mathrm{S}\}$

entonces

$$
J(u+v)=J(u)+|| v||_{a}^{2} \text { para todo } v \in S
$$

Demostración.

Del Teorema 2.1., a (u, v) $=(f, v)$. Entonces

$$
\begin{aligned}
J(u+v)-J(u) & =a(u+v, u+v)-2(f, u+v)-a(u, u)+2(f, u) \\
& =2 a(u, v)+a(v, v)-2(f, v) \\
& =a(v, v) \\
& =\|v\|_{a}^{2}
\end{aligned}
$$


Teorema 2.2.

Si u e X satisface la ecuación $L u=f$, entonces

1) $\mathrm{a}(\mathrm{u}, \mathrm{v})=(\mathrm{f}, \mathrm{v})$ para todo $\mathrm{v} \in \mathrm{K}$

2) $\mathrm{J}(\mathrm{u})=\min \{\mathrm{J}(\mathrm{v}): \mathrm{v} \in \mathrm{K}\}$

3) $\min \{\mathrm{J}(\mathrm{v}): \mathrm{v} \in \mathrm{K}\}=\min \{\mathrm{J}(\mathrm{v}): \mathrm{v} \in \mathrm{x}\}$

Demostración.

1) $\mathrm{a}(\mathrm{u}, \mathrm{v})=(\mathrm{Lu}, \mathrm{v})=(\mathrm{f}, \mathrm{v}), \mathrm{v} \in \mathrm{K}$

2) Se sigue de 1) y teorema 2.1 .

3) Conclusión inmediata de 2) y teorema 1.1 .

Observación.

Antes de probar el siguiente teorema es conveniente observar que si a (...) es elíptico en $\mathrm{K}$, entonces también es elíptico en $\mathrm{H}$. En efecto, para $v \in H$ existe una sucesión $\left(v_{n}\right)$ de Cauchy de elementos en $K$ tal que, por definición,

$$
\left\|v_{n}\right\|_{a} \rightarrow|| v \|_{a} \text { cuando } n \rightarrow \infty
$$

Tenemos $a\left(v_{n}, v_{n}\right) \geqslant \alpha \quad\left\|v_{n}\right\|_{2}^{2}$ para todo $n$.

Entonces, $\left(\left\|v_{n}\right\|_{2}\right)$ es una sucesión de Cauchy en $\mathbb{R} y$, por lo tanto, convergente. Por definición, $\|v\|_{2}=\underset{n \rightarrow \infty}{\lim _{n}}|| v_{n} \|_{2}$. Se sigue así que

$$
a(v, v) \geqslant \alpha \quad\|v\|_{2}^{2}
$$

Teorema 2.3 .

Existe un único $u \in H$ tal que

$$
J(u)=\min \{J(v): v e H\} \text { si a }(., \text { ) es elíptico en } H .
$$

Demostración.

$\mathrm{H}$ es un espacio de Hilbert para el producto interior a $(., \ldots)$.

Además,

$$
I(v)=(f, v)
$$

es una funcional lineal acotada en $\mathrm{H}$. 
En efecto, para $v \in H$ tenemos

$$
|I(v)|=|(f, v)| \leqslant\left.|| f||_{2}|| v\right|_{2} \leqslant\left.\frac{1}{\sqrt{\alpha}}|| f\left|\|_{2}\right||v|\right|_{a}
$$

Aplicando el teorema de representación de Riesz, existe un único u $\in \mathrm{H}$ tal que

$$
I(v)=(f, v)=a(u, v) \text { para todo } v \in H \text {. }
$$

Aplicando ahora el teorema 2.1, existe un único u $\in \mathrm{H}$ que minimiza la funcional $\mathrm{J}$ en $\mathrm{H}$.

Teorema 2.4 .

$$
\begin{aligned}
& \text { Si } a(\ldots) \text { es elíptico en } \mathrm{H} \text {, entonces } \\
& \quad \min \{\mathrm{J}(\mathrm{v}): \mathrm{v} \in \mathrm{H}\}=\min \{\mathrm{J}(\mathrm{v}): \mathrm{v} \in \mathrm{K}\} .
\end{aligned}
$$

Demostración.

\section{Basta probar que}

$$
\min \{\mathrm{J}(\mathrm{v}): \mathrm{v} \in \mathrm{H}\} \geqslant \min \{\mathrm{J}(\mathrm{v}): \mathrm{v} \in \mathrm{K}\} .
$$

Supongamos que existe $\mathrm{v} \in \mathrm{H}-\mathrm{K}$ tal que

$$
J(v)<\min \{J(v): v \in K\}
$$

Puesto que $H=\bar{K}$ en la norma $\|\cdot\| \|_{a}$, dado $\varepsilon, 0<\varepsilon<1$, existe $w \in k$ tal que

$$
|| v-w||_{a}<\varepsilon
$$

Entonces,

$$
\begin{aligned}
J(v) & =J(w+(v-w)) \\
& =a(w+(v-w), w+(v-w))-2(f, w+(v-w)) \\
& =J(w)+2 a(w, v-w)+a(v-w, v-w)-2(f, v-w)
\end{aligned}
$$

Luego,

$$
\begin{aligned}
|J(v)-J(w)| & \leqslant 2|| w||_{a}|| v-\left.w\right|_{a}+|| v-w||_{a}^{2}+2|| f||_{2}|| v-\left.w\right|_{2} \\
& \leqslant\left. 2|| w\right|_{a}|| v-\left.w\right|_{a}+|| v-w||_{a}^{2}+\frac{2}{\sqrt{\alpha}}|| f||_{2}|| v-\left.w\right|_{a} \\
& =\left(\left.2|| w\right|_{a}+|| v-\left.w\right|_{a}+\left.\frac{2}{\sqrt{\alpha}}|| f\right|_{2}\right)|| v-\left.w\right|_{a} \\
& <\left(2|| w||_{a}+1+\left.\frac{2}{\sqrt{\alpha}}|| f\right|_{2}\right) \varepsilon
\end{aligned}
$$


Como $\varepsilon$ es arbitrariamente pequeño, debe cumplirse que $J(v)=J(w)$. Entonces, $J(v) \geqslant \min \{J(w): w e k\}$,

resultado que contradice la hipótesis en el elemento v. Con lo cual, el teorema ha quedado demostrado. $\#$

\section{Teorema 2.5 .}

$\mathrm{Si}$ a $(.$, ) es elíptico en $\mathrm{H}$ y $\mathrm{si}$ u $\mathrm{E} \mathrm{X}$ es tal que Lu $=\mathrm{f}$ entonces

$$
J(u)=\min \{J(v): v \in H\}
$$

Demostración.

Se sigue en forma inmediata de los teoremas 2.2. y 2.4.

\#

\section{3.- EL METODO DE RITZ.}

Sea $\mathrm{S}$ un subespacio de $\mathrm{H}$ y $\mathrm{a}(.,$.$) elíptico en \mathrm{H}$. Tenemos $(f, v) \leqslant|(f, v)| \leqslant \frac{1}{\sqrt{\alpha}}|| f\left\|_{2}|| v\right\|_{a}$ para todo $v \in H$.

Entonces, para cualquier $\mathrm{v} \in \mathrm{H}$,

$$
\begin{aligned}
J(v) & =a(v, v)-2(f, v) \geqslant a(v, v)-\left.\frac{2}{\sqrt{\alpha}}|| f||_{2}|| v\right|_{a}= \\
& =\left(\left.|| v\right|_{a}-\left.\frac{1}{\sqrt{\alpha}}|| f\right|_{2}\right)^{2}-\left.\frac{1}{\alpha}|| f\right|_{2} ^{2} \\
& >-\left.\frac{1}{\alpha}|| f\right|_{2} ^{2} .
\end{aligned}
$$

Esto significa que la funcional $\mathrm{J}$ está acotada inferiormente cualquiera sea el subespacio $\mathrm{S}$. Por lo tanto,

$$
\mathrm{d}=\inf \{\mathrm{J}(\mathrm{v}): \mathrm{v} \in \mathrm{S}\} \text { existe }
$$


Por definición de ínfimo, existe una sucesión $\left(v_{n}\right)$ de elementos en $\mathrm{s}$ tal que

$$
\lim _{n \rightarrow \infty} J\left(v_{n}\right)=d
$$

Definición.

Cualquier sucesión de elementos en $\mathrm{s}$ tal que (3.1) sea cierta se llama sucesión minimizante para la funcional J en S.

\section{Teorema 3.1.}

Si a (.,.) es elíptico en $\mathrm{H}$ y si $\mathrm{S}$ es un subespacio cerrado de $\mathrm{H}$,

entonces existe un único u $\in \mathrm{S}$ tal que

$J(u)=\min \{J(v): v \in s\}$. En particular, cualquier sucesión mi nimizante para la funcional $J$ en $S$ converge a este elemento u en la norma $\mid 1 \cdot \|_{a}$

Demostración.

Sea $\left(v_{n}\right)$ una sucesión minizante para la funcional $J$ en $S$. Entonces, dado $\varepsilon>0$ existe un natural $N$ tal que

$$
\mathrm{d} \leqslant \mathrm{J}\left(\mathrm{v}_{\mathrm{n}}\right)<\mathrm{d}+\varepsilon \text { para todo } \mathrm{n} \geqslant \mathrm{N}
$$

Tenemos

$$
\begin{aligned}
& \left.\left\|\frac{v_{n}+v_{m}}{2}\right\|\right|_{a} ^{2}=\left.\frac{1}{4}|| v_{n}\right|_{a} ^{2}+\frac{1}{4}|| v_{m}||_{a}^{2}+\frac{1}{2} a\left(v_{n}, v_{m}\right) \\
& \left\|\frac{v_{n}-w_{m}}{2}\right\|_{a}^{2}=\frac{1}{4}|| v_{n}||_{a}^{2}+\frac{1}{4}|| v_{m}||_{a}^{2}-\frac{1}{2} a\left(v_{n}, v_{m}\right),
\end{aligned}
$$

Sumando miembro a miembro,

$$
\| \frac{v_{n}+v_{m}}{2}||_{a}^{2}+|| \frac{v_{n}-v_{m}}{2}||_{a}^{2}=\frac{1}{2}\left(|| v_{n}||_{a}^{2}+|| v_{m}||_{a}^{2}\right)
$$


De donde podemos escribir

$$
\| \frac{v_{n}-v_{m}}{2}||_{a}^{2}=\frac{1}{2}\left(J\left(v_{n}\right)+J\left(v_{m}\right)\right)-J\left(\frac{v_{n}+v_{m}}{2}\right)
$$

Por lo tanto, para $n, m \geqslant N$, tenemos

$$
0 \leqslant|| \frac{\mathrm{v}_{\mathrm{n}}-\mathrm{v}_{\mathrm{m}}}{2}||_{\mathrm{a}}^{2}<\frac{1}{2}(\mathrm{~d}+\varepsilon+\mathrm{d}+\varepsilon)-\mathrm{d}=\varepsilon .
$$

Dado que $\varepsilon>0$ es arbitrario, la sucesión $\left(v_{n}\right)$ es de Cauchy en la norma $\|.\|_{a}$. Luego, existe $u \in H$ tal que

$$
|| v_{n}-u||_{a} \rightarrow 0 \text { cuando } n \rightarrow \infty
$$

Como $\mathrm{S}$ es cerrado, $\mathrm{u} \in \mathrm{S}$. Entonces,

$$
\begin{aligned}
|| v_{n} \|_{a} & \left.\rightarrow|| u\right|_{a} \quad y \\
\left(f, v_{n}\right) & \rightarrow(f, u) .
\end{aligned}
$$

Por 10 tanto,

$$
J\left(v_{n}\right) \rightarrow J(u)
$$

Es decir,

$$
\begin{aligned}
& J(u)=\min \{J(v): v \in s\} \\
& \text { Sean } u_{1} \text { y } u_{2} \in S \text { tales que } J\left(u_{1}\right)=J\left(u_{2}\right)=d .
\end{aligned}
$$

Entonces, del teorema 2.1, tenemos

$$
\begin{aligned}
& a\left(u_{1}, v\right)=(f, v) \\
& a\left(u_{2}, v\right)=(f, v)
\end{aligned}
$$

para todo $\mathrm{v} \in \mathrm{S}$. Luego,

$$
a\left(u_{1}-u_{2}, v\right)=0 \text {, para todo } v \in S
$$




$$
\begin{aligned}
& \text { Para } v=u_{1}-u_{2} \text {, resulta } \\
& \quad a\left(u_{1}-u_{2}, u_{1}-u_{2}\right)=0
\end{aligned}
$$

De donde, $u_{1}=u_{2}$. Con lo cual, el teorema queda probado.

\section{\#}

El método de Ritz busca una aproximación de $u \in X$, solución de $\mathrm{Lu}=\mathrm{f}$, minimizando la funcional $\mathrm{J}$ sobre subespacios finito-dimensionales. Más precisamente, si $\mathrm{K}_{\mathrm{N}}$ es un subespacio $\mathrm{N}$-dimensional entonces el método de Ritz busca $v_{N} \in K_{N}$ tal que

$$
J\left(v_{N}\right)=\min \left\{J(v): v \in K_{N}\right\}
$$

Observamos que tal elemento $\mathrm{v}_{\mathrm{N}}$ existe $\mathrm{y}$ es único en virtud del teorema 3.1., pues todo subespacio finito-dimensional es cerrado. Sea

$$
\phi_{1}, \phi_{2}, \phi_{3}, \cdots, \phi_{\mathrm{N}}
$$

una base de $\mathrm{K}_{\mathrm{N}}$. Entonces

$$
v_{N}=\sum_{i=1}^{N} \alpha_{i} \phi_{i}
$$

Usando el teorema 2.1 , este elemento $v_{N}$ que minimiza la funcional J sobre $\mathrm{K}_{\mathrm{N}}$ debe satisfacer la ecuación

$$
a\left(v_{N}, v\right)=(f, v) \text { para todo } v \in K_{N}
$$

Entonces,

$$
\sum_{i=1}^{N} a\left(\phi_{i}, v\right) \alpha_{i}=(f, v) \text { para todo } v \in K_{N}
$$

En particular, para $v=\phi_{j}$

tenemos

$$
\sum_{i=1}^{N} a\left(\phi_{i}, \phi_{j}\right) \alpha_{i}=\left(f, \phi_{j}\right), j=1,2, \ldots, N
$$


Vemos así que la aproximación de Ritz puede ser caracterizada en términos de la solución de un sistema de ecuaciones lineales. La matriz de coeficientes

$$
A=\left(a\left(\phi_{i}, \phi_{j}\right)\right)
$$

es definida positiva. En efecto, si

$$
\begin{aligned}
v=\left(\alpha_{1}, \alpha_{2}, \ldots, \alpha_{N}\right)^{T} \text { entonces } \\
v^{T} A V=\sum_{i, j=1}^{N} a\left(\phi_{i}, \phi_{j}\right) \alpha_{i} \alpha_{j}= \\
=a\left(\sum_{i=1}^{N} \alpha_{i} \phi_{i}, \sum_{j=1}^{N} \alpha_{j} \phi_{j}\right) \\
\geqslant\left.\alpha|| \sum_{i=1}^{N} \alpha_{i} \phi_{i}\right|_{2} ^{2}>0
\end{aligned}
$$

para todo $\mathrm{V} \neq 0$ pues $\phi_{1}, \phi_{2}, \ldots, \phi_{\mathrm{N}}$ son linealmente independientes.

Hemos probado de este modo el siguiente teorema

Teorema 3.2.

Los coeficientes $\alpha_{1}, \alpha_{2}, \ldots, \alpha_{N}$ del único elemento $v_{N}$ que mini miza la funcional $\mathrm{J}$ sobre $\mathrm{K}_{\mathrm{N}}$ satisfacen el sistema de ecuaciones

$$
\sum_{i=1}^{N} a\left(\phi_{i}, \phi_{j}\right) \alpha_{i}=\left(f, \phi_{j}\right), j=1,2, \ldots, N
$$

cuya matriz de coeficiente es definida positiva cualquiera sea la base escogida para $\mathrm{K}_{\mathrm{N}}$.

Desde el punto de vista computacional es deseable tener una matriz A esparcida. Usualmente $a(.$,$) será dado por una integral y la$ matriz A será esparcida si el soporte de las funciones base es pequeño. Por ejemplo, si 


$$
a(u, v)=\int \frac{\int}{G}\left(\frac{\partial u}{\partial x} \frac{\partial v}{\partial x}+\frac{\partial u}{\partial y} \frac{\partial v}{\partial y}\right) d x d y
$$

entonces $a\left(\phi_{i}, \phi_{j}\right)=0$ cuando

$$
\text { (soporte } \phi_{i} \text { ) } \cap \text { (soporte } \phi_{j} \text { ) }=\phi \text {. }
$$

Teorema 3.3.

Si u minimiza la funcional $\mathrm{J}$ sobre $\mathrm{H}$ y $\mathrm{u}_{\mathrm{N}}$ lo hace en $\mathrm{K}_{\mathrm{N}}$ entonces

$$
|| u-\left.u_{N}\right|_{a}=\min \left\{|| u-v i v||_{a}: \stackrel{\sim}{v} \in k_{N}\right\}
$$

Demostración:

Del corolario del teorema 2.1. tenemos

$$
J(u+v)=J(u)+\|\left. v\right|_{a} ^{2} \text { para todo } v \in H
$$

En particular, para $\mathrm{v}=\mathrm{u}_{\mathrm{N}}-\mathrm{u}$, tenemos

$$
J\left(u_{N}\right)=J(u)+|| u_{N}-\left.u\right|_{a} ^{2}
$$

$$
\begin{aligned}
& \text { Luego, }\left\|\mathrm{u}-\mathrm{u}_{\mathrm{N}}\right\|_{\mathrm{a}}^{2}=\mathrm{J}\left(\mathrm{u}_{\mathrm{N}}\right)-\mathrm{J}(\mathrm{u}) \\
& \leqslant J(\tilde{v})-J(u)= \\
& =|| u-v \|_{a}^{2} \text { para todo } \stackrel{\sim}{v} \in K_{N}
\end{aligned}
$$

Entonces,

Luego

$$
|| u-u_{N} \|_{a} \leqslant \min \left\{|| u-\left.v\right|_{a}: \stackrel{\sim}{v} \in K_{N}\right\}
$$

$$
\left\|\mathrm{u}-\mathrm{u}_{\mathrm{N}} \mid\right\|_{\mathrm{a}}=\min \left\{|| \mathrm{u}-\tilde{v} \|_{\mathrm{a}}: \tilde{v} \in \mathrm{K}_{\mathrm{N}}\right\}
$$


4. APLICACION A UN PROBLEMA ELIPTICO DE SEGUNDO ORDEN EN UNA VARIABLE.

En esta sección consideramos el problema de aproximar la solución de :

$$
u \in C^{2}(a, b) \cap C^{0}[a, b]
$$

tal que

$$
\begin{aligned}
& \text { (4.1.) }-\left(p u^{\prime}\right)^{\prime}+o u=f, \quad x \in(a, b) \\
& \text { (4.2.) } u(a)=u(b)=0
\end{aligned}
$$

donde las funciones reales $p, \sigma$ y $f$ satisfacen las condiciones

1) $\mathrm{p} \in \mathrm{C}^{1}[\mathrm{a}, \mathrm{b}]$ con $\mathrm{p}(\mathrm{x}) \geqslant \ell>0$

2) $\sigma, f \in C^{0}[a, b], \sigma(x) \geqslant 0$.

$$
\text { Sea } x=\left\{u \in C^{2}(a, b) \cap C^{0}[a, b]: u(a)=u(b)=0\right\} \text {. }
$$

Claramente, $\mathrm{X}$ es un subespacio vectorial. Consideremos en este subespa cio el operador L correspondiente al primer miembro de (4.1), es decir,

$$
L u=-\left(p u^{\prime}\right)^{\prime}+\sigma u \text {. }
$$

Este operador es lineal y además satisface las propiedades

$$
(L u, v)=(u, L v)
$$

$$
(\mathrm{Iv}, \mathrm{v})>0, \quad \mathrm{v} \neq 0
$$

En efecto, para $u, v$ e $x$ tenemos

$$
\begin{aligned}
(L u, v) & =\int_{a}^{b}\left[-\left(p u^{\prime}\right)^{\prime}+\sigma u\right] v d x= \\
& =-\int_{a}^{b}\left(p u^{\prime}\right)^{\prime} v d x+\int_{a}^{b} \sigma u v d x \\
& =-\left.p u^{\prime} v\right|_{a} ^{b}+\int_{a}^{b} p u^{\prime} v^{\prime} d x+\int_{a}^{b} \sigma u v d x \\
& =\int_{a}^{b}\left(p u^{\prime} v^{\prime}+\sigma u v\right) d x
\end{aligned}
$$


Entonces,
$(4.5) \quad(L u, v)=\int_{a}^{b}\left(p u^{\prime} v^{\prime}+\sigma u v\right) d x$

Analogamente,

$$
(u, L v)=\int_{a}^{b}\left(p u^{\prime} v^{\prime}+\sigma u v\right) d x
$$

Por 10 tanto, $(L u, v)=(u, L v), u y v \in x$.

Pasamos ahora a probar (4.4). Sea $v$ e X, no idénticamente nula. Tenemos de (4.5)

$$
(L v, v)=\int_{a}^{b}\left(p\left(v^{\prime}\right)^{2}+o v^{2}\right) d x \geqslant 0
$$

Si $(L v, v)=0$ entonces $p\left(v^{\prime}\right)^{2}+\sigma v^{2}=0$ para todo $x$ e $(a, b)$. De esta igualdad, usando las hipótesis en las funciones p y $\sigma$ y la continuidad de v y de su derivada, se sigue que v debe ser idénticamente nula en $[a, b]$. Lo que contradice la elección de v. Con lo cual (4.4) queda probado.

Las propiedades de $\mathrm{L}$ nos permiten ver que $(\mathrm{Lu}, \mathrm{v})$ es un producto interior en $\mathrm{X}$.

Sea $K$ el subespacio vectorial de todas las funciones $v \in C^{\circ}[a, b]$ tales que

1) $v(a)=v(b)=0$

2) $v$ es absolutamente continua en $[a, b]$

3) $v^{\prime} \in L^{2}(a, b)$.

Observamos que la integral

$$
\int_{a}^{b}\left(p u^{\prime} v^{\prime}+\sigma u v\right) d x
$$

que aparece en el segundo miembro de (4.5), existe para todo $u, v \in K$. 
Esto nos permite definir

$$
a(u, v)=\int_{a}^{b}\left(p u^{\prime} v^{\prime}+\sigma u v\right) d x .
$$

Puede ser probado fácilmente que $a(.$,$) es un producto interior$ en $\mathrm{K}$. Sea

$$
\begin{aligned}
& \qquad|| v \|_{a}=\sqrt{a(v, v)}=\left(\int_{a}^{b}\left(p u^{\prime} v^{\prime}+\sigma u v\right) d x\right)^{1 / 2} \\
& \text { Es claro que si } u \in X y w \in K \text { entonces } \\
& (L u, w)=\int_{a}^{b}\left(p u^{\prime} w^{\prime}+\sigma u w\right) d x=a(u, w) .
\end{aligned}
$$

Con 10 cual queda probado que la restricción de a (.,.) a $\mathrm{x} \times \mathrm{K}$ satisface $(1.6)$.

Sea $\mathrm{H}$ la completación de $\mathrm{K}$ respecto de $\|\cdot\|_{\mathrm{a}}$,

Para u y $v \in K$ definimos

(4.7)

$$
(u, v)_{1}=\int_{a}^{b}\left(u^{\prime} v^{\prime}+u v\right) d x
$$

Puede ser probado que también $(.,)_{1}$ es un producto interior en K. La norma inducida por este nuevo producto interior será denotada por

$$
\|v\|_{1}=\sqrt{(v, v)_{1}}, v \in K
$$

El siguiente teorema nos muestra, en particular, la equivalencia de las normas $\|\cdot\|_{a} y\left\|_{1}\right\|_{1}$.

Teorema 4.1.

Existen constantes $\beta$ y $\gamma$ tales que para todo $v$ e $K$ se tiene

1) $\beta\|v\|_{a} \leqslant\|v\|_{1} \leqslant \gamma \quad\|v\|_{a}$

2) ||$v||_{2} \leqslant|| v||_{1}$

3) ||$v\left\|_{2} \leqslant \gamma \quad|| v\right\|_{a}$ 
Demostración:

La desigualdad 2) es inmediata. En efecto,

$$
\|v\|_{2}^{2}=\int_{a}^{b} v^{2} d x \leqslant \int_{a}^{b}\left(\left(v^{\prime}\right)^{2}+v^{2}\right) d x=\|v\|_{1}^{2}
$$

Sea $M=\operatorname{máx}_{x \in[a, b]}\{p(x), \sigma(x)\}$. Claramente,

$$
|| v||_{a}^{2} \leqslant M|| v||_{1}^{2} \text { para } v \in K
$$

Entonces, para $\beta=\frac{1}{\sqrt{M}}$, obtenemos

$$
\beta|| v||_{a} \leqslant|| v||_{1} \text { para todo } \mathrm{v} \in \mathrm{K} \text {. }
$$

La desigualdad 3) se sigue de 1) y 2). Luego, sólo falta probar que exis te $\gamma>0$ tal que

$$
|| v \|_{1} \leqslant\left.\gamma|| v\right|_{a}
$$

Probaremos primeramente esta desigualdad para $v$ en $C_{0}^{1}(a, b)$, el conjunto de todas las funciones reales con primera derivada continua y soporte compacto contenido en $(a, b)$. Sea $[-c, c]$ un intervalo cerrado que con tenga al intervalo $[a, b]$. Sea $\widetilde{v}$ la extensión de $v \in C_{0}^{1}(a, b)$ al intervalo $[-c, c]$ que se anula fuera de $(a, b)$. Entonces

$$
\tilde{v}(t)=\int_{-c}^{t} \tilde{v}^{\prime}(x) d x
$$

Aplicando la desigualdad de Cauchy-Schwartz tenemos

$$
\left.v^{2}(t) \leqslant(t+c) \int_{-c}^{t} \tilde{v}^{\prime}(x)\right)^{2} d x \leqslant 2 c \int_{c}^{c} \tilde{\left(v^{\prime}(x)\right)^{2}} d x
$$

Por 10 tanto,

$$
\int_{-C}^{c} \tilde{v}^{2}(x) d x \leqslant 4 c^{2} \int_{-c}^{c}\left(\tilde{v}^{\prime}\right)^{2} d x
$$


Luego, $\int_{a}^{b} v^{2} d x \leqslant 4 c^{2} \int_{a}^{b}\left(v^{\prime}\right)^{2} d x$

Sea $\delta=4 c^{2} \quad$ y $m=\min _{x \in[a, b]}\{p(x)\}$

Entonces,

$$
\begin{aligned}
\|\left. v\right|_{1} ^{2} & =\int_{a}^{b}\left(\left(v^{\prime}\right)^{2}+v^{2}\right) d x \leqslant(1+\delta) \int_{a}^{b}\left(v^{\prime}\right)^{2} d x \\
& \leqslant \frac{1+\delta}{m} \int_{a}^{b} p\left(v^{\prime}\right)^{2} d x \\
& \leqslant \frac{1+\delta}{m} \int_{a}^{b}\left(p\left(v^{\prime}\right)^{2}+o v^{2}\right) d x
\end{aligned}
$$

En consecuencia, para $v \in C_{0}^{1}(a, b)$ tenemos

$$
\|v\|_{1} \leqslant \gamma\|v\|_{a}, \quad \gamma^{2}=\frac{1+\delta}{m}
$$

Este resultado puede ser extendido a $K$ haciendo uso del hecho que $C_{0}^{1}(a, b)$ es denso en $\mathrm{K}$ respecto de la norma $\|.\|_{1}$. En efecto, para $\mathrm{v} \in \mathrm{K}$ existe una sucesión $\left(v_{n}\right)$ en $C_{0}^{1}(a, b)$ tal que

$$
|| v_{n}-v \|_{1} \rightarrow 0 \text { cuando } n \rightarrow \infty
$$

Sabemos que

$$
|| v_{n}-v\left\|_{a} \leqslant \sqrt{M} \quad\right\| v_{n}-v \|_{1}
$$

Entonces, ||$v_{n}-\left.v\right|_{a} \rightarrow 0 \quad$ cuando $n \rightarrow \infty$

En particular,

$$
\left\|v_{n}\right\|_{1} \rightarrow\|v\|_{1} \text { y }\left\|v_{n}\right\|_{a} \rightarrow\|v\|_{a}
$$

cuando $\mathrm{n} \rightarrow \infty$. De la desigualdad

$$
\left\|v_{n}\right\|_{1} \leqslant \gamma|| v_{n} \|_{a}
$$

tomando límite cuando $\mathrm{n} \rightarrow \infty$, se sigue que 


$$
|| v\left\|_{1} \leqslant \gamma|| v \mid\right\|_{a} \text { en } \mathrm{K} \text {. }
$$

Queda así probado el teorema 4.1.

\section{Observación.}

1) El punto 1) del teorema 4.1. nos dice que las normas $\|$.$\| y y\|\cdot\| \|_{1}$ son equivalentes en $\mathrm{K}$. Por lo tanto, $\mathrm{H}$ es también la completación de $\mathrm{K}$ respecto de la norma $\|\cdot\|_{1}$. Entonces, el teorema 4.1. es váli do en $\mathrm{H}$.

2) For su parte, el punto 3) del teorema 4.1. nos asegura que a (.,.) es elíptico en $\mathrm{K} y$ entonces en $\mathrm{H}$. En efecto, para $\mathrm{V} \in \mathrm{K}$

$$
a(v, v)=|| v||_{a}^{2} \geqslant \frac{1}{\gamma^{2}}|| v||_{2}^{2}=a|| v||_{2}^{2},
$$

donde $\alpha=\frac{1}{\gamma^{2}}$, para todo $\mathrm{v} \in \mathrm{K}$.

Podemos, en consecuencia, aplicar en este problema toda la teoría elaborada en las secciones 2 y 3 . En particular, podemos utilizar el método de Ritz para aproximar su solución mediante el uso de funciones seccionalmente lineales, como se muestra a continuación.

Sea

$$
\Delta: \mathrm{x}_{0}=\mathrm{a}<\mathrm{x}_{1}<\mathrm{x}_{2}<\ldots \ldots<\mathrm{x}_{\mathrm{m}}<\mathrm{x}_{\mathrm{m}+1}=\mathrm{b}
$$

una partición de $[\mathrm{a}, \mathrm{b}]$. Consideremos las funciones $\ell_{i}$ para $i=1,2, \ldots$, $n$, definidas como sigue

$$
\ell_{i}(x)=\left\{\begin{array}{l}
\frac{x-x_{i-1}}{x_{i}-x_{i-1}}, x_{i-1} \leqslant x \leqslant x_{i} \\
\frac{x_{i+1}-x}{x_{i+1}-x_{i}}, x_{i} \leqslant x \leqslant x_{i+1} \\
0, \quad x \notin\left[x_{i-1}, x_{i+1}\right] .
\end{array}\right.
$$


La siguiente figura ilustra la gráfica de $\ell_{i}$

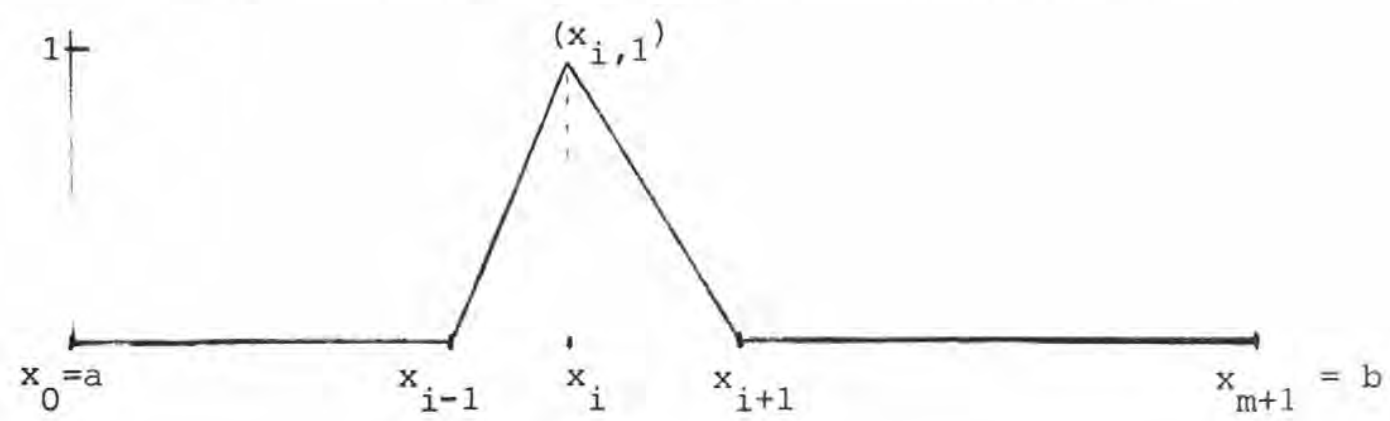

Claramente, $\ell_{i} \in \mathrm{K}$ y de (4.9) tenemos

$$
\ell_{i}\left(x_{j}\right)=\delta_{i j} .
$$

Además, $\left\{l_{1}, l_{2}, l_{3}, \ldots . l_{m}\right\}$ es un conjunto linealmente independientes de funciones. Sea $K_{m}$ el subespacio generado por este conjunto de funcio nes. Los coeficientes de la aproximación de Ritz en $\mathrm{K}_{\mathrm{m}}$ están dados por la solución del sistema

$$
\sum_{i=1}^{n} a\left(\ell_{i}, \ell_{j}\right) \alpha_{i}=\left(f, \ell_{j}\right), j=1,2, \ldots, m
$$

Es fácil ver que para estas funciones base se tiene

$$
a\left(\ell_{i}, \ell_{j}\right)=0 \text { si }|i-j| \geqslant 2
$$

Esto implica que la matriz en (4.10) resulta ser tridiagonal. Esto, como es sabido, representa desde ya una ventaja desde el punto de vista computacional.

En particular para $x_{i+1}-x_{i}=h$, es decir, para una partición en subintervalos de igual longitud, tenemos

$$
a\left(\ell_{i}, \ell_{i}\right)=\int_{x_{i-1}}^{x_{i}}\left(p \ell_{i} \ell_{i}^{\prime}+\sigma \ell_{i} \ell_{i}\right) d x+\int_{x_{i}}^{x_{i+1}}\left(p \ell_{i}^{\prime} \ell_{i}^{\prime}+\sigma \ell_{i} \ell_{i}\right) d x
$$




$$
\begin{aligned}
& =\frac{1}{h^{2}} \int_{x_{i-1}}^{x_{i}}\left(p+\sigma\left(x-x_{i-1}\right)^{2}\right) d x+\frac{1}{h^{2}} \int_{x_{i}}^{x_{i+1}}\left(p+\sigma\left(x_{i+1}-x\right)^{2}\right) d x \\
& a\left(l_{i}, l_{i+1}\right)=\int_{x_{i}}^{x_{i+1}}\left(p \ell_{i} \ell_{i+1}^{\prime}+\sigma l_{i+1}{ }_{i+1}\right) d x= \\
& =-\frac{1}{h^{2}} \int_{x_{i}}^{x_{i+1}}\left(p-\sigma\left(x_{i+1}-x\right)\left(x-x_{i}\right)\right) d x \\
& a\left(\ell_{i}, f\right)=\int_{x_{i-1}}^{x_{i}} \ell_{i} f d x+\int_{x_{i}}^{x_{i+1}} \ell_{i} f d x \\
& =\frac{1}{h}\left[\int_{x_{i-1}}^{x_{i}}\left(x-x_{i-1}\right) f d x+\int_{x_{i}}^{x_{i+1}}\left(x_{i+1}-x\right) f d x\right]
\end{aligned}
$$

Si $p(x) \equiv 1$ y $\sigma(x) \equiv c$ (constante) entonces

$$
\begin{aligned}
& a\left(l_{i}, l_{i}\right)=\frac{2}{h}+\frac{2}{3} c h=\frac{2}{h} \quad(\text { si c }=0) \\
& a\left(l_{i}, l_{i+1}\right)=-\frac{1}{h}+\frac{1}{6} c h=-\frac{1}{h} \quad(\text { si c }=0)
\end{aligned}
$$

El segundo miembro debe ser obtenido mediante integración numé rica, como también los términos $a\left(l_{i}, l_{i}\right)$ y $a\left(l_{i}, l_{i+1}\right)$ cuando $p$ y $\sigma$ no son constantes, Para $\mathrm{p}(\mathrm{x}) \equiv 1$, el uso de la fórmula de simpson para aproximar las integrales conduce a

$$
\begin{aligned}
& a\left(l_{i}, l_{i}\right)=\frac{2}{h}+\frac{h}{6}\left[\sigma\left(x_{i}-h / 2\right)+2 \sigma\left(x_{i}\right)+\sigma\left(x_{i}+h / 2\right)\right] \\
& a\left(l_{i}, l_{i+1}\right)=-\frac{1}{h}+\frac{h}{6} \sigma\left(x_{i}+h / 2\right) \\
& a\left(l_{i}, f\right)=\frac{h}{3}\left[f\left(x_{i}-h / 2\right)+f\left(x_{i}\right)+f\left(x_{i}+h / 2\right)\right] .
\end{aligned}
$$


Es interesante señalar que si

$$
\begin{aligned}
& v(x)=\sum_{j=1}^{m} \alpha_{j} \ell_{j}(x) \quad \text { entonces } \\
& v\left(x_{i}\right)=\alpha_{i}, \text { para } i=1,2,3, \ldots, m .
\end{aligned}
$$

Esto significa que los valores $\alpha_{1}, \alpha_{2}, \ldots, \alpha_{m}$ obtenidos al resolver el sistema (4.10) son los valores de la aproximación de Ritz en los puntos $\mathrm{x}_{1}, \mathrm{x}_{2}, \mathrm{x}_{3}, \ldots, \mathrm{x}_{\mathrm{m}}$ de la partición $\Delta$.

Ejemplo 1:

$$
\begin{gathered}
-u^{\prime \prime}+\left(1+x^{2}\right) u=\left(2+x^{2}\right) \text { sen } x, \quad 0<x<\pi \\
u(0)=u(\pi)=0
\end{gathered}
$$

Se puede verificar que $u=\operatorname{sen} x$ es la solución para este problema. Los siguientes fueron los resultados obtenidos al aplicar el método de Ritz, con el uso de la fórmula de simpson en la integración numérica, para los valores de $h$ que se indican. 
Para $h=\frac{\pi}{101}$

\subsection{5}

0.3355350

0.6077666

0.8216698

0.9567152

0.9999416

0.9471997

0.8035508

0.5827811

0.3060786
0.0621730

0.3646714

0.6321718

0.8390014

0.9653099

0. 9989744

0.9367634

0.7846470

0.5572240

0.2763211
0.0931843 0.3934551

0.6559654 0.8555215 9.9729708 0.9970408 0.9254209 0.7649840 0.5311278 0. 2462962
0.1241055 0.4218582 0.6791245 0. 8712138 0.9796904 0.9941427 0.9131831 0.7445811 0.5045177 0.2160330
0.1549066 0.4498531 0.7016265 0. 8860633 0.9854622 0.9902828 0.9000618 0. 7234577 0.4774196 0.1855608
0.1855578 0.4774128 0.7234498 0. 9000556 0.9902806 0.9854648 0.8860698 0.7016345 0.4498596 0.1549091
0.2160295 0.5045107 0.7445732 0. 9131772 0.9941410 0.9796935 0.8712205 0.6791324 0.4218643 0.1241075
0.2462923 0.5311205 0. 7649762 0.9254154 0.9970396 0.9729743 0. 8555284 0.6559733 0.3934610 0.0931858
Para $h=\frac{\pi}{201}$

\subsection{4}

0.1710845

0.3223688

0.4657939

0.5978633

0.7153572

0.8154112

0.8955860

0.9539270

0.9890119

0.9999852

0.9865795

0.9491215

0.8885245

0.8062656

0.7043505

0.5852636

0.4519082

0.3075355

0.1556653
0.0312550

0.1864626

0.3371244

0.4795674

0.6103189

0.7261912

0.8243595

0.9024304

0.9585007

0.9912033

0.9997410

0.9839055

0.9440830

0. 8812443

0.7979212

0.6931697

0.5725189

0.4379105

0.2926260

0.1402073
0.0468729

0.2017951

0.3517977

0.4932238

0.6226254

0.7368478

0.8331064

0.9090544

0.9628402

0.9931526

0.9992525

0.9809912

0.9388139

0.8737488

0.7873821

0.6818195

0.5596345

0.4238058

0.2776449

0.1247151
0.0624794

0.2170783

0.3663851

0.5067596

0.6347798

0.7473244

0.8416498

0.9154563

0.9669445

0.9948593

0.9985199

0.9778372

0.9333154

0.8660399

0.7776507

0.6703028

0.5466133

0.4095975

0.2625960

0.1091925
0.0780706

0.2323085

0. 3808830

0.5201717

0.6467791

0.7576184

0.8499875

0.9216345

0.9708126

0.9963229

0.9975434

0.9744444

0.9275890

0.8581194

0.7677293

0.6586224

0.5334585

0.3952892

0.2474830

0.0936431
0.0936428

o. 2474820

0. 3952878

0.5334567

0.6586204

0.7677274

0.8581177

0.9275876

0.9744435

0.9975431

0.9963232

0.9708135

0.9216359

0.8499893

0.7576204

0.6467810

0.5201735

$0.38-8844$

0.2323095

0.0780709
0.1091920

0.2625950

0.4095960

0.5466114

0.6703008

0.7776488

0.8660382

0.9333141

0.9778364

0.9985197

0.9948597

0.9669455

0.9154577

0.8416515

0.7473264

0.6347817

0.5067614

0.3663865

0. 2170792

0.0624796
0.1247147 0.2776438

0.4238042

0.5596326

0.6818175

0. 7873802

0.8737471

0.9388126

0.9809905

0.9992524

0.9931530

0.9628412

0.9090559

0.8331082

0.7368498

0.6226273

0.4932255

0.3517991

0.2017959

0.0468731
0.2763167 0.5572165 0.7846393

0.9367583

0.9989737

0.9653139

0.8390087

0.6321797

0.3646769

0.0621740

0.3060739

0.5827735

0.8035432

0.9471949

0.9999413

0.9567196

0.8216772

0.6077744

0.3355401 


\section{APLICACION A UN PROBLEMA ELIPTICO DE SEGUNDO ORDEN EN EL PLANO.}

\subsection{FORMULACION DEL PROBLEMA.}

Aquí consideramos el problema de aproximar la solución de: Encontrar u $e C^{2}(G) \cap C^{0}(G)$ tal que

$$
\begin{aligned}
& -\left(p u_{x}\right)_{x}-\left(q u_{y}\right)_{y}+\sigma u=f, \quad(x, y) \in G \\
& u(x, y)=0,(x, y) \in \partial G,
\end{aligned}
$$

donde $G$ es una región acotada en $\mathbb{R}^{2}$, en la cual el teorema de Green es aplicable, y donde las funciones p, q, $\sigma$, f satisfacen las condiciones

1) $\mathrm{p}, \mathrm{q} \in \mathrm{C}^{1}(\overline{\mathrm{G}}) \operatorname{con} \mathrm{p}(\mathrm{x}, \mathrm{y}) \geqslant \ell>0 \mathrm{y} \mathrm{q}(\mathrm{x}, \mathrm{y}) \geqslant \ell>0$.

2) $\sigma$, $f \in C^{0}(\bar{G}), \sigma(x, y) \geqslant 0$

$$
\text { Sea } x=\left\{u \in C^{2}(G) \cap C^{0}(\bar{G}): u(x, y)=0,(x, y) \in \quad \partial G\right\}
$$

En este subespacio vectorial está definido el operador lineal l correspondiente al primer miembro de (5.1),

$$
L u=-\left(p u_{x}\right)_{x}-q\left(q u_{y}\right)_{y}+\sigma u
$$

Tenemos que $L$ satisface para $u, v \in X$

$$
(\mathrm{Lu}, \mathrm{v})=(\mathrm{u}, \mathrm{Lv})
$$

$$
(\mathrm{Lu}, \mathrm{u})>0 \text { si } u \neq 0,
$$

donde $(f, g)=\iint_{\bar{G}} f g d x d y$

En efecto, aplicando el teorema de Green tenemos

$$
\begin{aligned}
& (L u, v)=\iint\left[-\left(p u_{x}\right)_{x}-\left(q u_{y}\right) y+\sigma u\right] v d x d y \\
& =\iint_{\bar{G}}\left[-\left(p u_{x} v\right) x-\left(q u_{y} v\right){ }_{y}^{\bar{G}}\right] d x d y+\iint_{\bar{G}}\left[p u_{x} v_{x}+q u_{y} v_{y}+\sigma u v\right] d x d y
\end{aligned}
$$


$=\iint_{\partial G} q u v d x+p u_{x} v d y+\iint_{\frac{G}{}}\left[p u_{x} v v_{x}+q u v_{y} v_{y}+\sigma u v\right] d x d y$.

La primera integral de este segundo miembro es cero, pues $\mathrm{v}$ se anula en la frontera de G. Entonces,

Análogamente,

$$
(L u, v)=\iint_{G}\left[p u_{x} v_{x}+q u v_{y} v+\sigma u v\right] d x d y
$$

$$
(u, L v)=\iint_{\frac{G}{G}}\left[p u v_{x} x+q u v_{y}\right] d x d y
$$

Con 10 cual, (5.3) se sigue. Pasamos ahora a probar (5.4). Sea u $\in$ X, u no identicamente nula. Tenemos de (5.5),

Luego, si $(L u, u)=0$ entorices

$$
(L u, u)=\iint_{\bar{G}}\left[p u_{x}^{2}+q u_{y}^{2}+\sigma u^{2}\right] d x d y
$$

$$
p u_{x}^{2}+q u_{y}^{2}+\sigma u^{2}=0 \text { para todo }(x, y) \in G
$$

de donde se sigue, usando las hipótesis en p, q y $\sigma$ y la continuidad de u y de sus derivadas parciales junto con las condiciones de frontera, que $u$ debe ser idénticamente nula. Lo que contradice la elección de u $\in$ X. Queda así probado (5.4).

Las propiedades del operador l recién probadas nos permiten ver que $(\mathrm{Lu}, \mathrm{V})$ es un producto interior en $\mathrm{X}$.

Sea $K$ el subespacio vectorial de las funciones $v \in C^{0}(\bar{G})$ tales que

1) $\mathrm{v}(\mathrm{x}, \mathrm{y})=0,(\mathrm{x}, \mathrm{y}) \in \partial \mathrm{G}$

2) v es absolutamente continua como función de $x$ con y fijo, y también como función de $y$ con $x$ fijo.

3) $\mathrm{v}_{\mathrm{x}}, \mathrm{v}_{\mathrm{y}} \in \mathrm{L}^{2}(\mathrm{G})$

La integral

$$
\iint_{\bar{G}}\left[p u_{x} v_{x}+q u v_{y}+\sigma u v\right] d x d y
$$


del segundo miembro de (5.5), existe para u y v en $\mathrm{K}$. Podemos entonces definir

$$
a(u, v)=\iint_{\bar{G}}\left[p u_{x} v_{x}+q u v_{y} y+\sigma u v\right] d x d y
$$

Fácilmente se prueba que $a(.$,$) es un producto interior en \mathrm{K}$. Sea

$$
\|v\|_{a}=\sqrt{a(v, v)}, v \in K
$$

la norma inducida por este producto interior.

También puede ser probado que $s i \mathrm{u} \in \mathrm{X} \mathrm{y} v \in \mathrm{K}$ entonces

$$
(L u, v)=\int \frac{\int}{G}\left[p u_{x} v_{x}+q u v_{y} y+o u v\right] d x d y=a(u, v)
$$

Con esto queda probado que $a(.$, ) satisface $(1.6)$.

Sea $\mathrm{H}$ la completación de $\mathrm{K}$ respecto de $\|\cdot\|_{\mathrm{a}}{ }$. Para u y v en $\mathrm{K}$ definimos

$$
(u, v)_{1}=\iint_{\bar{G}}\left[u_{x} v_{x}+u_{y} v_{y}+\sigma u v\right] d x d y
$$

Puede ser probado que $(.,)_{1}$ es otro producto interior en $K$, cuya norma inducida denotaremos mediante

$$
|| v \|_{1}=\sqrt{(v, v)_{1}}, v \in K .
$$

Teorema 5.1 .

Existen constantes $\beta$ y $\gamma$ tales que para todo $v \in K$ tenemos
1) $\beta\|v\|_{a} \leqslant\|v\|_{1} \leqslant \gamma|| v \|_{a}$
2) ||$v||_{2} \leqslant|| v||_{1}$
3) ||$v \|_{2} \leqslant r|| v||_{a}$ 
Demostración:

La demostración es completamente análoga a la del teorema 4.1, ahora con $[-c, c] \times[-c, c]$ en lugar de $[-c, c]$

Observación:

1) E1 punto 1) del teorema 5.1 nos dice que las normas $\|\cdot\|$ a $y\|\cdot\| \|_{1}$ son equivalentes en $\mathrm{K}$. Por lo tanto, H es también la completación de $\mathrm{K}$ respecto de la norma $\|.\|_{1}$. Luego, el teorema 5.1 tambiế, se cumple para $\mathrm{H}$.

2) El punto 3) del teorema 5.1 nos permite concluir que a (.,.) es elípti co en $\mathrm{K} y$ entonces en $\mathrm{H}$. En efecto, para $\mathrm{V} \in \mathrm{K}$

$$
a(v, v)=|| v||_{a}^{2} \geqslant \frac{1}{\gamma^{2}}|| v||_{2}^{2}=\alpha|| v||_{2}^{2},
$$

donde $\alpha=\frac{1}{\gamma^{2}}$

En consecuencia, podemos aplicar al problema 5.1) - 5.2) toda

la teoría elaborada en las primeras secciones.

$$
\begin{gathered}
\text { Consideremos aquí, en particular, el caso } \\
\qquad \begin{aligned}
p(x, y)= & q(x, y)=1 \\
\sigma(x, y) & =0
\end{aligned}
\end{gathered}
$$

para todo $(x, y) \in G=[a, b] x[a, b]$,

es decir, la ecuación de poisson en un cuadrado con condiciones homogéneas en la frontera

$$
\begin{aligned}
-\left(\frac{\partial^{2} u}{\partial x^{2}}+\frac{\partial^{2} u}{\partial y^{2}}\right) & =f, \quad(x, y) \in \text { int } G \\
u(x, y) & =0, \quad(x, y) \text { e } \partial G .
\end{aligned}
$$

En este caso,

$$
a(u, v)=\int_{a}^{b} \int_{a}^{b}\left(\frac{\partial u}{\partial x} \frac{\partial v}{\partial x}+\frac{\partial u}{\partial y} \frac{\partial v}{\partial y}\right) d x d y
$$




\subsection{SUBDIVISION DEI DOMINIO EN RECTANGULOS.}

Sea $\Delta$ una partición uniforme de $[a, b]$ dada por

$$
\Delta: a=x_{0}<x_{1}<x_{2}<\cdots<x_{m}<x_{m+1}=b
$$

Entonces, $\Delta x \Delta$ es una partición de $[a, b] \times[a, b]$ en rectángulos. Consideremos como funciones base para el método de Ritz, las funciones $\phi_{i j}, 1 \leqslant i, j \leqslant m$ dadas por

$$
\phi_{i j}(x, y)=\ell_{i}(x) \ell_{j}(y),
$$

donde las funciones $\ell_{i}$ están dadas por

$$
\ell_{i}(x) \begin{cases}\frac{x-x_{i-1}}{h}, & x_{i-1} \leqslant x \leqslant x_{i} \\ h & , x_{i} \leqslant x \leqslant x_{i+1} \\ 0, & x \notin\left[x_{i-1}, x_{i+1}\right],\end{cases}
$$

con $h=x_{i+1}-x_{i}=\frac{b-a}{m+1}, y$ similarmente para $\ell_{j}(Y)$.

Fácilmente puede ser probado que el conjunto de funciones

$$
\left\{\phi_{i j}: 1 \leqslant i \leqslant m, 1 \leqslant j \leqslant m\right\}
$$

es linealmente independiente y que si

$\phi(x, y)=\sum_{i=1}^{m} \sum_{j=1}^{m} \alpha_{i j} \ell_{i}(x) l_{j}(y)$ entonces

$\alpha_{i j}=\phi\left(x_{i}, y_{j}\right)$.

La función $\phi$ es la aproximación de Ritz para el conjunto de funciones base $\phi_{i j}$ si y sólo si los coeficientes $\alpha_{i j}$ satisfacen el sis tema de ecuaciones 
(5.10) $\sum_{i=1}^{\mathrm{m}} \sum_{j=1}^{\mathrm{m}} a\left(\phi_{i j}, \phi_{k \ell}\right) \alpha_{i j}=\left(f, \phi_{k \ell}\right), \quad 1 \leqslant k, l \leqslant m$

Para estas funciones base se tiene que si $|i-j| \geqslant 2$ б

$|k-\ell| \geqslant 2$ entonces a $\left(\phi_{i j}, \phi_{k l}\right)=0$.

Las otras entradas de la matriz de coeficientes del sistema de ecuaciones (5.10) están dadas por

$$
\begin{aligned}
& a\left(\phi_{i j}, \phi_{i j}\right)=\frac{8}{3}, a\left(\phi_{i j}, \phi_{(i+1)}(j+1)\right)=-\frac{1}{3} \\
& a\left(\phi_{i j}, \phi_{i(j+1)}\right)=-\frac{1}{3}, a\left(\phi_{i j}, \phi_{(i+1) j}\right)=-\frac{1}{3}
\end{aligned}
$$

para todo $(i, j)$.

En particular, si ordenamos el segundo miembro de (5.10) según

$$
\begin{aligned}
& \left(f, \phi_{11}\right), \quad\left(f, \phi_{21}\right), \ldots \ldots,\left(f, \phi_{m 1}\right), \\
& \left(f, \phi_{12}\right), \quad\left(f, \phi_{22}\right), \ldots \ldots,\left(f, \phi_{m 2}\right), \\
& \vdots \\
& \left(f, \phi_{1 m}\right), \quad\left(f, \phi_{2 m}\right), \ldots .,\left(f, \phi_{m m}\right),
\end{aligned}
$$

la matriz de coeficientes adopta la forma tridiagonal en bloques:

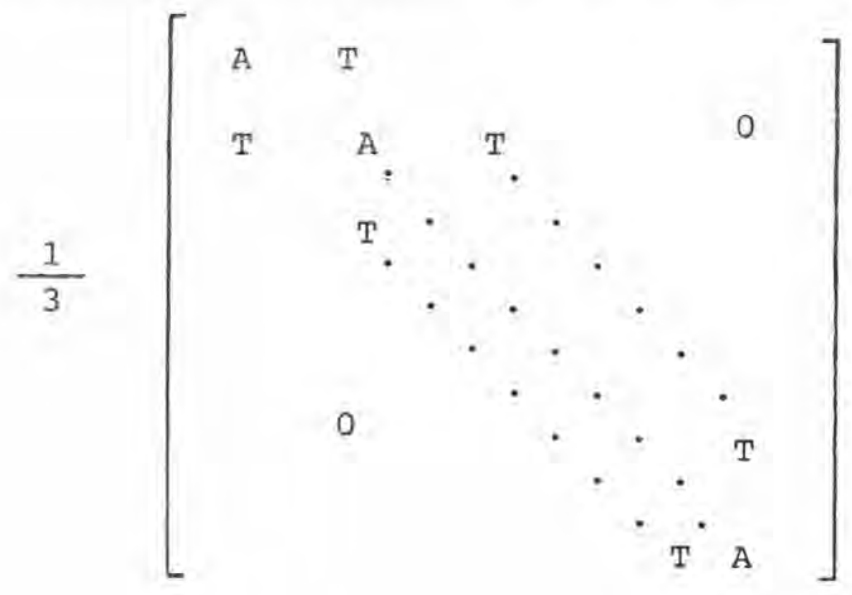


donde



de orden $m \times m$.

Puede probarse que las matrices A y $T$ conmutan. Esto implica que puede usarse el método de descomposición matricial expuesto en [6] en la resolución del sistema (5.10), previo conocimiento de los autovalores de A y T. Estos autovalores resultan ser

$$
\lambda_{k}=8-2 \cos \frac{k \pi}{m+1}, \quad 1 \leqslant k \leqslant m
$$

para la matriz A y

$$
\omega_{k}=-\left(1+2 \cos \frac{k \pi}{m+1}\right), \quad 1 \leqslant k \leqslant m
$$

para la matriz T.

El método de la descomposición matricial es particularmente eficiente si se emplea la transformada rápida de Fourier. El segundo miembro del sistema (5.10) puede ser aproximado mediante integración numérica. En particular, ( $f, \phi_{k} l^{\prime}$ es aproximado por

$$
\begin{aligned}
\left(f, \phi_{k \ell}\right) \approx \frac{h^{2}}{9} & {\left[f\left(x_{k-1}+\frac{h}{2}, y_{l-1}+\frac{h}{2}\right)+f\left(x_{k}, y_{l-1}+\frac{h}{2}\right)+f\left(x_{k}+\frac{h}{2}, y_{l-1}+\frac{h}{2}\right)+\right.} \\
+ & f\left(x_{k-1}+\frac{h}{2}, y_{\ell}\right)+f\left(x_{k}, y_{l}\right)+f\left(x_{k}+\frac{h}{2}, y_{l}\right)+ \\
+ & \left.f\left(x_{k-1}+\frac{h}{2}, y_{\ell}+\frac{h}{2}\right)+f\left(x_{k}, y_{\ell}+\frac{h}{2}\right)+f\left(x_{k}+\frac{h}{2}, y_{\ell}+\frac{h}{2}\right)\right]
\end{aligned}
$$


que resulta de aplicar la fórmula de Simpson para aproximar el valor de la integral

$$
\begin{aligned}
\left(f, \phi_{k l}\right) & =\int_{a}^{b} \int_{a}^{b} f(x, y) \ell_{k}(x) \ell_{j}(y) d x d y= \\
& =\int_{x_{k-1}}^{x} \int_{l-1}^{y_{\ell+1}} f(x, y) \ell_{k}(x) \ell_{j}(y) d x d y .
\end{aligned}
$$

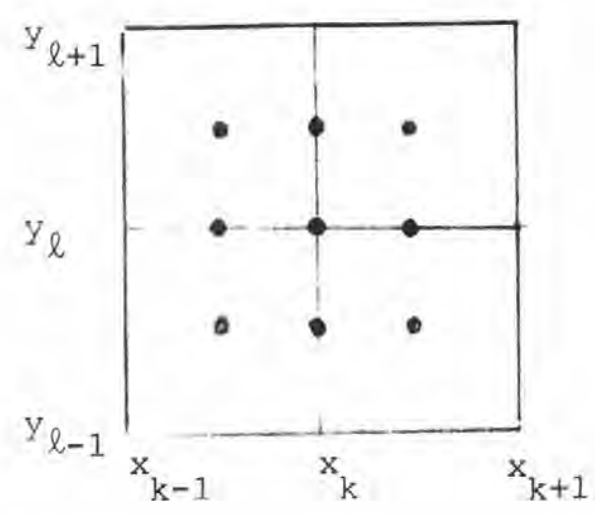

Ejemplo 2.

$$
\begin{gathered}
-\left(\frac{\partial^{2} u}{\partial x^{2}} \frac{\partial^{2} u}{\partial y^{2}}\right)=2 \pi^{2} \text { sen } \pi x \operatorname{sen} \pi y,(x, y) \in \text { int } G \\
u(x, y)=0,(x, y) \in \partial G
\end{gathered}
$$

donde $G=[0,1] \times[0,1]$.

La solución exacta es $u(x, y)=\operatorname{sen} \pi x$ sen $\pi y$.

Los resultados por nosotros obtenidos al aplicar el método de Ritz, aplicando el método de la descomposición matricial y usando la aproximación dada para el segundo miembro, se incluyen en las tablas siguientes para el valor de $m$ que se indica, el valor en la posición $(i, j)$ corresponde a la aproximación obtenida para

$$
u\left(\frac{i}{m+1}, \frac{j}{m+1}\right), 1 \leqslant i, j \leqslant m .
$$




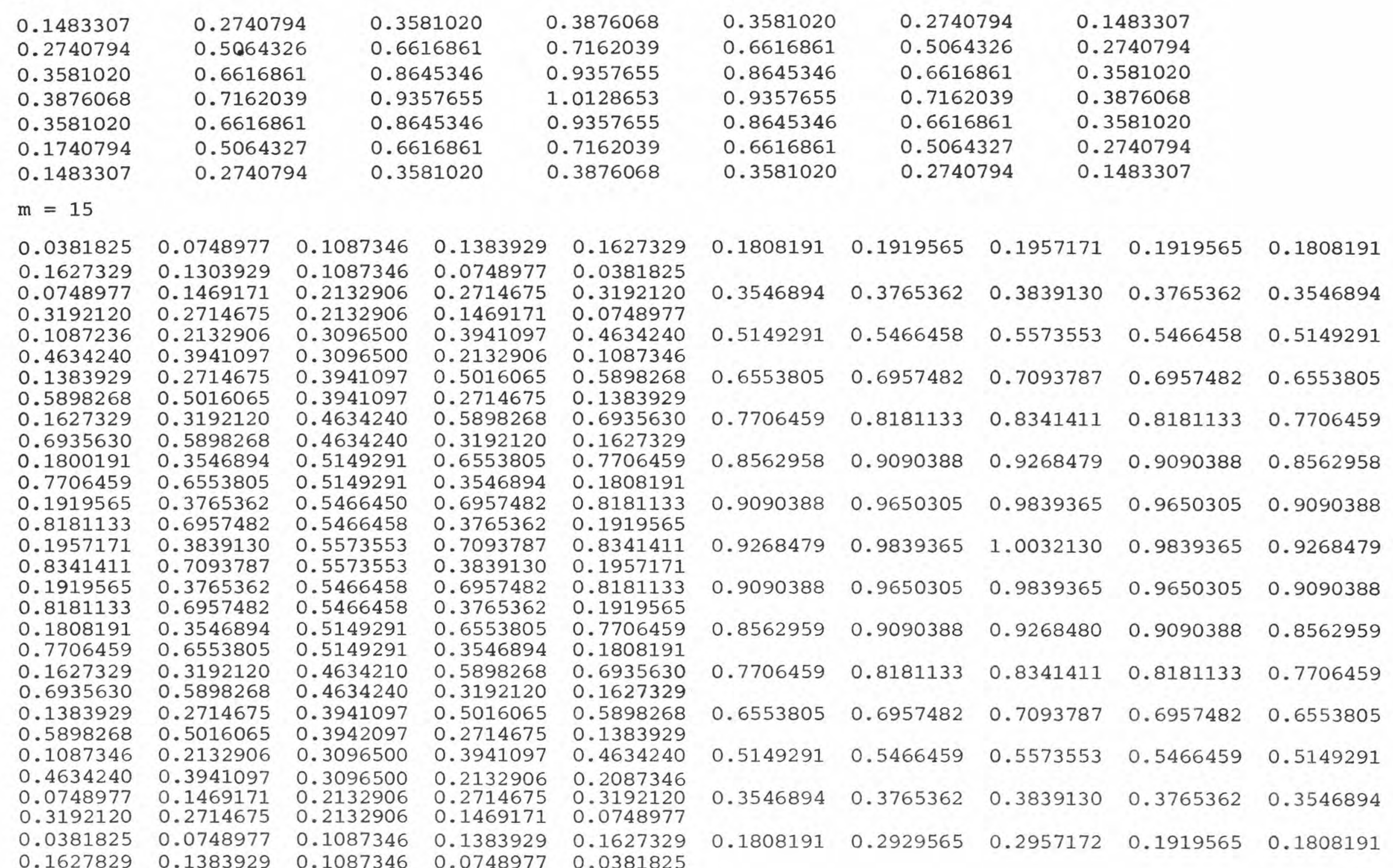




\subsection{SUBDIVISION DEL DOMINIO EN TRIANGULOS.}

Consideremos nuevamente el problema (5.1) - (5.2) con

$\mathrm{p}(\mathrm{x}, \mathrm{y})=\mathrm{q}(\mathrm{x}, \mathrm{y})=1$ y $\sigma(\mathrm{x}, \mathrm{y})=0$ para todo $(\mathrm{x}, \mathrm{y})$ en $\mathrm{G}=[\mathrm{a}, \mathrm{b}] \mathrm{x}[\mathrm{a}, \mathrm{b}]$, es decir, el mismo problema tratado en la sección 5.2.

A diferencia de la sección anterior, nos interesa considerar aquí una triangularización del dominio G, es decir una subdivisión del cuadrado $\mathrm{G}$ en triángulos, como se indica en la figura de abajo.

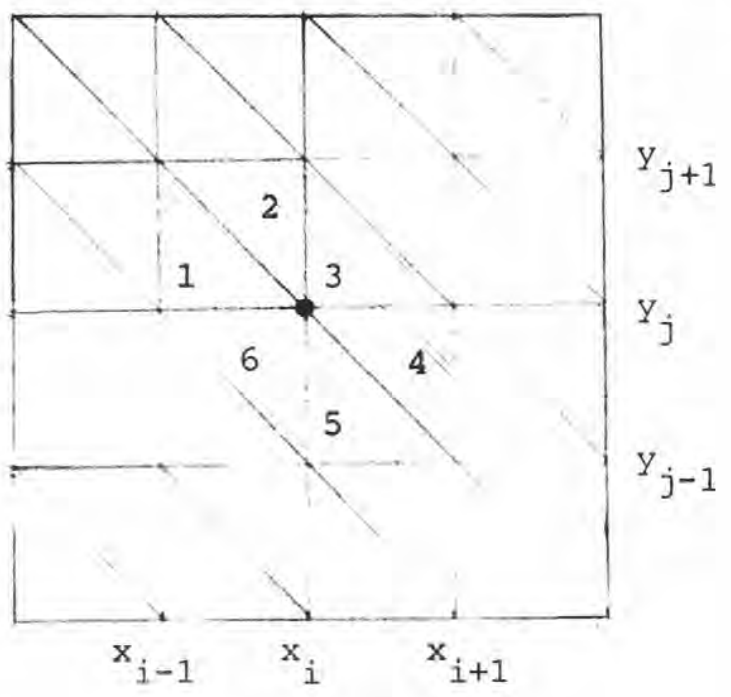

Estos triángulos o "elementos" serán denotados por $\Delta_{\ell} y$ deben satisfacer la siguiente condición: la intersección de dos triángulos distintos cualesquiera consiste exactamente de un vértice o de un lado - bien es vacía.

Denotemos por $\xi_{i j}$ los vértices de los triángulos $\Delta_{\ell}$ que no per tenecen a la frontera de G.

En la figura anterior hemos enumerado los triángulos o "elemen tos" que contienen el vértice $\xi_{i j}$. 
Elegimos una base de funciones $\psi_{i j}$ satisfaciendo las siguientes condiciones:

1) $\psi_{i j} \in c^{0}(\bar{G})$

2) La restricción de $\psi_{i j}$ al triángulo $\Delta_{\ell}$ es un polinomio de primer grado en $\mathbb{R}^{2}$.

3) $\psi_{i j}\left(\xi_{K \ell}\right)=\delta_{i K} \delta_{j \ell}$

4) $\psi_{i j}(x, y)=0$ para $(x, y)$ e $\partial G$

Vemos que estas funciones $\psi_{i j}$ pertenecen al espacio $\mathrm{K} y$ se anulan sobre cada triángulo $\Delta_{\ell}$ que no contiene el vértice $\xi_{i j}$. Estas funciones $\psi_{i j}$ pueden ser construidas del modo siguiente:

Consideremos para el triángulo $\Delta_{1}=1$ de la figura anterior, es decir, para el triángulo

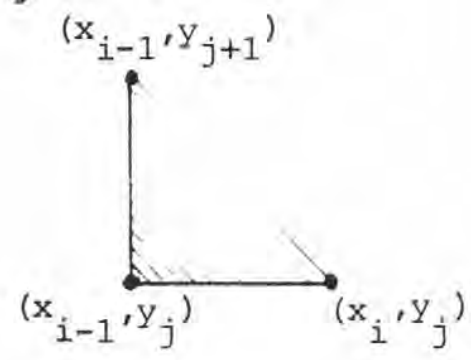

el polinomio lineal en $\mathbb{R}^{2}: u(x, y)=a+b x+c y$.

Imponiendo las condiciones $u\left(x_{i}, y_{j}\right)=1, u\left(x_{i-1}, y_{j}\right)=u\left(x_{i-1}, y_{j+1}\right)=0$

y resolviendo el sistema lineal correspondiente encontramos que

$$
u(x, y)=u_{1}(x, y)=1+\frac{x-x_{i}}{h},(x, y) \text { e } \Delta_{1}=1
$$

Del mismo modo se encuentra que

$$
\begin{aligned}
& u_{2}(x, y)=1-\frac{y-y_{j}}{h},(x, y) \in \Delta_{2}=2 \\
& u_{3}(x, y)=1+\frac{x_{i}+y_{j}}{h}-\frac{x+y}{h},(x, y) \in \Delta_{3}=3
\end{aligned}
$$




$$
\begin{aligned}
& u_{4}(x, y)=1-\frac{x-x_{i}}{h} ;(x, y) \text { e } \Delta_{4}=4 \\
& u_{5}(x, y)=1+\frac{y-y_{j}}{h} ;(x, y) \text { e } \Delta_{5}=5 \\
& u_{6}(x, y)=1-\frac{x_{i}+y_{j}}{h}+\frac{x+y}{h} ;(x, y) \in \Delta_{6}=6
\end{aligned}
$$

donde $h=x_{i}-x_{i-1}=y_{j}-y_{j-1}$.

Entonces podemos definir

$$
\psi_{i j}(x, y)=u_{\ell}(x, y) \text { para }(x, y) \in \Delta_{\ell} ; \ell=1,2, \ldots, 6
$$

Puede verse fácilmente que si

$$
\begin{aligned}
& \psi(x, y)=\sum_{i=1}^{m} \sum_{j=1}^{m} \alpha_{i j} \psi_{i j}(x, y) \text {, entonces } \\
& \psi\left(x_{i}, y_{j}\right)=\alpha_{i j} y \text { además que el conjunto de funciones }
\end{aligned}
$$

$\left\{\psi_{i j} ; i, j=1,2, \ldots, m\right\}$ es linealmente independiente.

La aproximación de Ritz a la solución de nuestro problema está dada ahora por (5.12), donde los coeficientes $\alpha_{i j}$ son obtenidos como la solución del sistema lineal

$$
\sum_{i=1}^{m} \sum_{j=1}^{m} a\left(\psi_{i j}, \psi_{K \ell}\right) \alpha_{i j}=\left(f, \psi_{K \ell}\right) ; k, \ell=1,2, \ldots, m .
$$

Las entradas de la matriz de coeficientes del sistema lineal

(5.13) están dadas por

$$
a\left(\psi_{i j} \psi_{K \ell}\right)=\iint_{G}\left[\frac{\partial \psi_{i j}}{\partial x} \frac{\partial \psi_{K \ell}}{\partial x}+\frac{\partial \psi_{i j}}{\partial y} \frac{\partial \psi_{K \ell}}{\partial y}\right] d x d y
$$

y puesto que las derivadas parciales en (5.14) toman los valores $\frac{1}{h}, \frac{-1}{h}$ 。 0 , se tiene que

$$
a\left(\psi_{i j}, \psi_{i j}\right)=4 ; a\left(\psi_{i j}, \psi_{i(j \pm 1)}\right)=-1
$$




$$
a\left(\psi_{i j}, \psi_{(i \pm 1) j}\right)=-1
$$

En todos los demás casos a $\left(\psi_{i j}, \psi_{K \ell}\right)=0$

Es interesante notar aquí que triangularizar el dominio G y utilizar las funciones base (5.11), nos lleva al conocido operador de diferencias de 5 puntos del método de Ias diferencias finitas

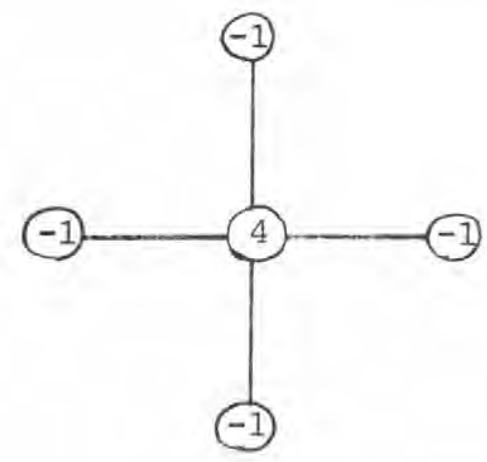

Una consecuencia de esto es que en ambos métodos, el de Ritz con la base de funciones definidas por (5.11) y el de las diferencias finitas, el miembro izquierdo del sistema lineal resultante es el mis mo. En efecto, obtenemos en este caso la matriz de coeficientes tridiagonal en bloques



e I es la matriz identidad. El segundo miembro de este sistema en cambio, está dado para el método de las diferencias finitas por $h^{2} f\left(x_{i}, y_{j}\right)$, mientras que para el método de Ritz es 
$(5.15)$

$$
\left(f, \psi_{K \ell}\right)=\iint_{G}\left[f(x, y) \psi_{K \ell}(x, y)\right] d x d y \quad y
$$

puede ser aproximado numéricamente como en la sección 5.2. Sin embargo, si aproximamos (5.14) por una fórmula conveniente, puede probarse que es te segundo miembro también coincide con el segundo miembro del sistema lineal resultante en el método de las diferencias finitas. En efecto, consideremos el triángulo $\Delta_{1}=1$

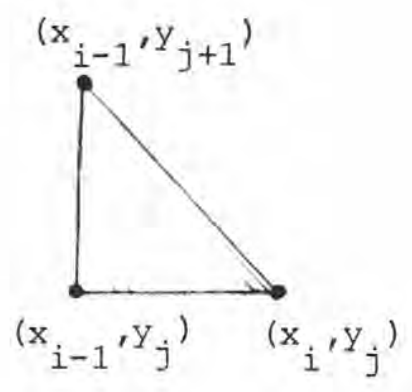

y la fórmula de aproximación

$$
\iint_{\bar{G}} g(x, y) d x d y \cong \frac{h^{2}}{6}\left[g\left(x_{i-1, j}\right)+g\left(x_{i}, y_{j}\right)+g\left(x_{i-1, j+1}\right)\right]
$$

Entonces tenemos para nuestro triángulo que

$$
\begin{gathered}
\iint_{1}\left[f(x, y) \psi_{i j}(x, y)\right] d x d y=\iint_{1}\left[f(x, y) u_{1}(x, y)\right] d x d y= \\
=\frac{h^{2}}{6} f\left(x_{i}, y_{j}\right)
\end{gathered}
$$

Lo mismo ocurre en los otros cinco triángulos. Así tenemos

$$
\iint_{\bar{G}}\left[f(x, y) \psi_{i j}(x, y)\right] d x d y=h^{2} f\left(x_{i}, y_{j}\right) .
$$


Ejemplo 3.

Consideremos el mismo problema del ejemplo 2, vale decir,

$$
\begin{aligned}
&-\left(\frac{\partial^{2} u}{\partial x^{2}}+\frac{\partial^{2} u}{\partial y^{2}}\right)=f(x, y),(x, y) e \text { Int } G \\
& u(x, y)=0,(x, y) \in \partial G
\end{aligned}
$$

donde $f(x, y)=2 \pi^{2}$ sen $\pi x$ sen $\pi y \quad y \quad G=[0,1] \times[0,1]$

Aplicando el método de Ritz con la base de funciones $\psi_{i j}$ definidas por (5.11) (dominio G triangularizado) obtuvimos los siguientes resultados para $\mathrm{m}=15$ : 


\begin{tabular}{|c|c|c|c|c|c|c|c|c|c|}
\hline 0.0381827 & 0.0748982 & 0.1087353 & 0.1383937 & 0.1627338 & 0.1808201 & 0.1919576 & 0.1957183 & 0.1919576 & 0.1808201 \\
\hline .1627338 & 0.1383937 & 0.1087353 & 0.0748982 & 0.0381827 & & & & & \\
\hline .0748982 & 0.1469180 & .2132919 & 0.2714691 & 0.3192139 & 0.3546915 & 0.3765385 & 0.3839153 & .3765385 & 0.3546915 \\
\hline . 3192139 & .2714691 & 0.2132919 & 0.1469180 & 0.0748982 & & & & & \\
\hline .1087353 & .2132919 & 0.3096518 & 0.3941120 & 0.4634267 & 0.5149322 & 66491 & 3586 & 5491 & 0.5149322 \\
\hline .4634267 & .3941120 & .3096518 & .2132919 & .1087353 & & & & & \\
\hline .1383937 & .2714691 & .3941120 & 6095 & 8303 & .6553844 & .6957523 & 0.7093829 & 0.6957523 & 0.6553844 \\
\hline .5898303 & 6095 & 120 & 591 & 3937 & & & & & \\
\hline .1627338 & 0.3192139 & 267 & 303 & 5671 & 0.7706505 & 0.8181182 & 0.8341461 & 0.8181182 & 0.7706505 \\
\hline 671 & .5898303 & 0.4634267 & 139 & 7338 & & & & & \\
\hline .1808201 & 0.3546915 & 0.5149322 & 844 & 6505 & 0.8563009 & 0.9090442 & 0.9268535 & 0.9090442 & 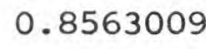 \\
\hline 505 & .6553844 & 0.51 & 915 & 0.1808201 & & & & & \\
\hline . 1919576 & 0.3765384 & 0.5466491 & 7523 & 31182 & 0 . & 362 & 0.983 & 0.9650362 & 0.9090442 \\
\hline 182 & .6957523 & 0.5466491 & 384 & 0.1919576 & & & & & \\
\hline 183 & 0.3839153 & 0.557 & 829 & 1461 & 0 & 4 & 0 & 4 & 35 \\
\hline 461 & 829 & 586 & 153 & 57183 & & & & & \\
\hline 576 & 385 & 91 & 523 & 1182 & 0.9090442 &  & 0.98 & 362 & .9090442 \\
\hline 182 & 523 & 491 & 0.3 & 576 & & & & & \\
\hline 201 & 6915 & 0.514 & 0.6 & 6505 & 0.8563010 & 9090442 & 9268535 & 442 & 3010 \\
\hline 05 & 14 & 22 & 5 & 8201 & & & & & \\
\hline 38 & 9 & 0. & 0 . & 671 & 0.7706505 & 0.8181182 & 0.8341461 & 0.8181182 & .7706505 \\
\hline 571 & 4 & 67 & 0. & 338 & & & & & \\
\hline 0.1 & 0.2714691 & 0.3941120 & 095 & 0.5898304 & 0.6553844 & 0.6957523 & 0.7093829 & 0.6957523 & 0.6553844 \\
\hline 0.5898301 & 0.5016095 & 0.3941120 & 0.2714691 & 0.1383937 & & & & & \\
\hline 0.1087353 & 0.2132919 & 0.3096518 & 0.3941120 & 0.4634267 & 0.5149322 & 0.3460491 & 0.5573586 & 0.5466491 & 0.5149322 \\
\hline 0.4634267 & 0.3941120 & 0.3096518 & 0.2132919 & 0.1087353 & & & & & \\
\hline 0.0748982 & 0.1469180 & 0.2132919 & 691 & 0.3192139 & 0.3546915 & 0.3765385 & 0.3839153 & 0.3765385 & 0.3546915 \\
\hline 0.3192139 & 0.2 & & & 982 & & & & & \\
\hline 0.0381827 & 0.0748982 & 0.1087353 & 0.1383937 & 0.1627338 & 0.1808201 & 0.1919576 & 0.1957183 & 0.191 & .18 \\
\hline .1627338 & .1383937 & 0.1087353 & 0.0748982 & .0381827 & & & & & \\
\hline
\end{tabular}




\section{COMENTIARIO.}

Queremos comentar finalmente que si la región $G$, en que está de finido nuestro problema de valor en la frontera, admite una partición re gular, como en el último ejemplo, entonces el método de Ritz lleva a fór mulas de diferencias finitas. La aplicación de estas fórmulas al proceso de discretización de nuestro problema resulta ser, desde un punto de vista práctico bastante más simple e implica por consiguiente un menor esfuerzo computacional. Sin embargo la importancia del método de Ritz o de elemento finito reside en su aplicabilidad a problemas con regiones que pueden ser particionadas en figuras geométricas arbitrarias.

\section{BIBLIOGRAFIA.}

[1] Mercier B., Topics in Finite Element Solution of Elliptic Problems, Tata Institute of Fundamental Research, 1979, Springer-Verlag.

[2] Meis T., Marcowitz U., Numerical Solution of Partial Differential Equations, 1978, Springer-Verlag.

[3] Mikhailov V.P., Partial Differential Equations, 1978, Mir Publishers, Moscow.

[4] Mitchell A.R., Wait R., The Finite Element Method in Partial Differential Equations, 1978, Wiley.

[5] Prenter P.M., Splines and Variational Methods, 1975, Wiley.

[6] Rojo, Soto, Valdivia, Aplicación del método de Descomposición Matricial y la Transformada Rápida de Fourier a la solución de la Ecuación de Poisson. Revista Proyecciones, $\mathrm{N}^{\circ} 4$, Julio 1983.

[7] Schultz, Spline Analysis, 1973, Prentice-Hall. 